\title{
Guest editorial: WWWJ special issue of the 21th international Conference on Web Information Systems Engineering (WISE 2020)
}

\author{
Hua Wang ${ }^{1} \cdot$ Zhisheng Huang $^{2}$
}

Published online: 5 November 2021

(c) The Author(s), under exclusive licence to Springer Science+Business Media, LLC, part of Springer Nature 2021

\section{WISE2020}

The 21th International Conference on Web Information Systems Engineering (WISE 2020) was held in Amsterdam, Netherlands, October 20-24, 2020. It was processed through online and off- line for participants as some countries have closed board due to the global pandemic situation. However, WISE2020 was very successful. Building on the success of its predecessors, WISE

2020 continues to be a major international forum for researchers, professionals, and industrial practitioners to share their knowledge in the rapidly growing area of Web technologies, methodologies, and applications. The first WISE event took place in Hong Kong, China (2000). Then the trip continued to Kyoto, Japan (2001); Singapore (2002); Rome, Italy (2003); Brisbane, Australia (2004); New York, USA (2005); Wuhan, China (2006); Nancy, France (2007); Auckland, New Zealand (2008); Poznan, Poland (2009); Hong Kong, China (2010); Sydney, Australia (2011); Paphos, Cyprus (2012); Nanjing, China (2013); Thessaloniki, Greece (2014); Miami, USA(2015); Shanghai, China (2016); Puschino, Russia (2017); Dubai, UAE (2018) and Hong Kong, China (2019). This year, for the first time, WISE was held in Amsterdam, Netherlands, supported by VU Amsterdam (Netherlands) and Victoria University (Australia).

A total of 190 research papers were submitted to the conference for consideration, and each paper was double-blind reviewed by at least three reviewers. Finally, 37 submissions were selected as full papers (with an acceptance rate of $19.5 \%$ approximately), plus 45 as short papers. The research papers cover the areas of social network data analysis, recommender systems, topic modeling, data diversity, data similarity, context-aware recommendation, prediction, big data processing, cloud computing, event detection, data mining, sentiment analysis, ranking in social networks, microblog data analysis, query processing, spatial and temporal data, graph theory and non-traditional environments. In addition to regular and short papers, WISE2020 program also featured an international workshop on artificial intelligence for mental health (AI4MH2020). We are honored to have several

Hua Wang

hua.wang@vu.edu.au

Victoria University, Footscray, Australia

2 Vrije University Amsterdam, Amsterdam, Netherlands 
of the world's leading experts in the field join us as distinguished keynote speakers and invited speakers.

AI4MH2020 is designed to promote the research and discussion on the applications of Artificial Intelligence Technology on Mental Health [3, 10]. The workshop focuses on the work of Chatbots for mental health and the work of AI for suicide prevention and rescue. In particular, it is interested in the relevant work such as Tree Hole Rescue through AI technology in real life applications [1,4]. Tree Hole Secret is based on an Indian myth. A person who has a secret should go to the forest and look for a tree with a hole. After whispering it into the hole, the secret would stay in the tree forever. Modern Tree hole is a metaphor of a social media in which the owner of the social media is dead and is used for people to express their painful and despair feeling. Many young people express their suicidal feelings and intentions through online social media, e.g., Twitter, Microblog. It provides the possibility of using Artificial Intelligence and big data technology to detect the posts where someone expressed the suicidal signal from internet social media [8]. "Tree-hole Rescue" has provided a successful example of AI applications as a case study for it.

\section{The special issue}

6 top ranked papers out of 37 full papers at WISE 2020 have been selected for the special issue of World Wide Web Journal (WWWJ). The selected papers underwent a rigorous extra refereeing and revision process. In particular, the six special issues papers have been extended with at least $60 \%$ new and unpublished material. Note that, adding more related work or extending the introduction was not considered in the $60 \%$; rather the new content often includes more technical and implementation details, improved algorithms, more experiment results, etc. The revised papers again went through the review and revision processes in accordance with WWWJ guidelines.

The paper titled "Joint service-function deployment and task scheduling in UAVFogassisted data- driven disaster response architecture" by Wei et al. aims to jointly optimize the deployment of service functions (SFs) and the task scheduling at UAVFog nodes to minimize the task response latency. After introducing the collaboration structure between UAVFog nodes, joint SF deployment and task scheduling is formulated as an optimization problem. Then, three algorithms are put forward to tackle the problem: 1) Dependency and topology-aware SF deployment (DeToSFD) algorithm is developed to determine the initial deployment location of each SF; 2) Context-aware greedy task scheduling (CoGTS) algorithm is put forward to schedule an arrived task; 3) Congestion-aware SF reallocation (CoSFR) algorithm is developed to reallocate SFs in case of congestion at an instance of an SF. Finally, a series of experiments are conducted to evaluate the performance of the proposed algorithms.

The paper "Graph embedding based real-time social event matching for EBSNs recommendation" by Wu et al. defines an EBSN as a heterogeneous information network, and performs graph embedding to represent the nodes in it, which can more effectively reflect the hidden features of nodes and contribute to mining users and events preferences. Then heuristic social event matching methods are employed to effectively find overall optimal recommendations between users and events under spatio-temporal constraints in real-time. This forms a two-stage framework, i.e., the representation learning stage and the real-time matching stage. Authors conducted experiments on the Meetup dataset to verify the effectiveness of the framework by combining different graph embedding methods and heuristic 
matching algorithms. The results show that the proposed framework yields improvements in the matching success rate, user satisfaction, and user waiting time.

The paper by Zong et al., presents a problem of copyright infringement in the music industry, especially for Internet-based platforms. The existing audio watermarking methods for copyright protection have limited robustness against the desynchronization attacks $[2,9]$. Besides, most of the methods are not designed for stereo signals, which are widely used by online-based music and video platforms. In this paper, authors present a desynchronization-attack-resilient watermarking method for stereo signals. In the watermark embedding process, both the left channel and the right channel of the host signal are firstly segmented and transformed into the frequency domain using the discrete cosine transform (DCT). Secondly, the linear correlation between channels (LCBC) in the DCT domain is calculated using the Pearson correlation coefficient (PCC). LCBC is robust against both desynchronization attacks and other common attacks. Theoretical analysis and experimental results demonstrate the effectiveness of the proposed method.

The paper "Charge prediction modeling with interpretation enhancement driven by double-layer criminal system" by Li et al. studies AI methods to predict legal judgments that has become a hot spot in recent years. Charge prediction is one of the core tasks of Legal Judgment Prediction (LJP). This paper aims to predict charge from complicated legal facts, so as to help the court make judgments or provide legal professional guidance to non-professionals. However, how to add the legal theory framework to the modeling to improve the interpretability is a challenge, which has few researches at present. To address this problem, authors use Double-layer Criminal System as a guide to build Charge Prediction modeling called DCSCP which aims to predict charges in the criminal law of China. It is supposed to achieve multi-granularity inference of legal charges by obtaining the subjective and objective elements from the fact descriptions of legal cases. Experimental results show that our DCSCP can provide interpretable predictions, and it can maintain performance compared to other state-of-the-art charge prediction models.

The paper by Yin et al. proposes an integrated consecutive batch learning framework to predict the probable exploitation time of vulnerabilities. To achieve a better performance, authors combine features extracted from both vulnerability descriptions and the Common Vulnerability Scoring System in the proposed framework [6, 7]. In particular, an Adaptive Sliding Window Weighted Learning (ASWWL) algorithm is designed to tackle the dynamic multiclass imbalance problem existing in many industrial applications including exploitation time prediction. A series of experiments are carried out on a real-world dataset, containing 24,413 exploited vulnerabilities disclosed between 1990 and 2020. Experimental results demonstrate the proposed ASWWL algorithm can significantly enhance the performance of the minority classes without compromising the performance of the majority class. Besides, the proposed framework achieves the most robust and state-of-the-art performance compared with the other five consecutive batch learning algorithms.

The paper titled "Exploiting intra- and inter-session dependencies for session-based recommendations" by Wang et al. aims to predict an item via learning the dynamic and short-term preferences of users [5]. In order to effectively extract both intra- and inter-session dependencies from not only the session information but also the side information, to further improve the accuracy of next-item recommendations, this paper proposes a novel hypergraph learning (HL) framework. The HL framework mainly contains three modules, i.e., a hypergraph construction module, a hypergraph learning module, and a next-item prediction module. The hypergraph construction module constructs a hypergraph to connect the users, items and item attributes together in a unified way. Then, the hypergraph learning module learns the informative latent representation for each item by extracting 
both intra- and inter-session dependencies embedded in the constructed hypergraph. Also, a latent representation for each user is learned. After that, the learned latent representations are fed into the next-item prediction module for next-item recommendations. The experimental results show that the HL framework outperforms the state-of-the-art approaches on two real-world datasets.

Acknowledgements We are very indebted to the reviewers who reviewed the papers very carefully. We would also like to thank all the authors who submitted their papers to the special issue. Special thanks to the journal editors for their great help and support in organizing the issue.

\section{References}

1. F Zhang, et al. Decision-based evasion attacks on tree ensemble classifiers. World Wide Web 23 (5), 2957-2977, 2020

2. H Wang, et al. Special issue on security and privacy in network computing. World Wide Web 23 (2), 951-957, 2020

3. Islam, M.R., et al.: Depression detection from social network data using machine learning techniques. Health information science and systems. 6(1), 1-12 (2018)

4. $\mathrm{J} \mathrm{He}$, et al. A framework for cardiac arrhythmia detection from IoT-based ECGs. World Wide Web 23 (5), 2835-2850, 2020

5. J Yin, et al. Adaptive Online Learning for Vulnerability Exploitation Time Prediction International Conference on Web Information Systems Engineering, 252-266, 2020

6. J Yin, et al. Vulnerability exploitation time prediction: an integrated framework for dynamic imbalanced learning. World Wide Web, 1-23, 2021

7. Li, H., et al.: Multi-window based ensemble learning for classification of imbalanced streaming data. World Wide Web. 20(6), 1507-1525 (2017)

8. R Singh, et al. Antisocial behaviour analyses using deep learning. International Conference on Health Information Science, 133-145, 2020

9. R.U. Rasool, et al. CyberPulse++: A machine learning-based security framework for detecting link flooding attacks in software defined networks. International Journal of Intelligent Systems, 36, 38523879. 2021

10. Sarki, R., et al.: Automated detection of mild and multi-class diabetic eye diseases using deep learning. Health Information Science and Systems. 8(1), 1-9 (2020)

Publisher's note Springer Nature remains neutral with regard to jurisdictional claims in published maps and institutional affiliations. 